\title{
REVISIÓN Y ANÁLISIS DE LAS \\ DISTINTAS INTERPRETACIONES DE LA \\ INTERCULTURALIDAD EN EL AULA MUSICAL
}

\section{María del Mar Bernabé Villodre}

\section{RESUMEN}

En este artículo se ofrece una revisión de las distintas propuestas de trabajo de la música en contextos educativos pluriculturales. Un análisis en profundidad permite comprobar que el uso de la terminología relacionada con la diversidad cultural es muy libre por parte del profesorado de música, lo que demuestra la necesidad de revisar su formación. Estas experiencias denominadas indistintamente multiculturales y/o interculturales sirven como excusa para presentar como modelo una breve síntesis de la experiencia propia en estos contextos, que sí se ajusta a los principios de la educación intercultural. Se ha intentado mostrar cómo esas interpretaciones de la terminología han llevado a usos que no favorecen el principal objetivo de la educación intercultural, como es garantizar la reconstrucción cultural compartida.

Palabras clave: interculturalidad, educación intercultural, música, educación musical.

\section{TITLE: REVIEW AND ANALYSIS OF THE DIFFERENT INTERPRETATIONS OF MULTICULTURALISM IN THE MUSIC CLASSROOM}

\section{ABSTRACT}

This article offers a review of the various proposals for working in multicultural music education contexts. A thorough analysis permits us to confirm that the terminology related to cultural diversity is used very liberally by music teachers, demonstrating the need to review their training. These experiences interchangeably called multicultural and/or intercultural serve as an excuse for presenting a brief summary of the author's own experience in these settings as a model which is adjusted to the principles of intercultural education. We have tried to show how these interpretations of terminology have led to applications that do not support the main aim of intercultural education, which is to ensure shared cultural reconstruction.

Keywords: intercultural, intercultural education, music, music education.

Correspondencia con la autora: María del Mar Bernabé Villodre. < elchesociologia@gmail.com> Universidad Católica San Antonio de Murcia (UCAM). Original recibido: 12-06-13. Original aceptado: 21-02-14 


\section{La importancia del conocimiento de la terminología relacionada con la diversidad cultural}

Cuando un país presencia un aumento de la diversidad cultural debe replantearse sus currículos educativos para que éstos reflejen esa pluriculturalidad. En España esta intención se fue dando de forma pausada, aunque con el tiempo y tras las diferentes reformas, se han podido observar importantes cambios. Éstos quedaron (y continúan quedando) plasmados en las materias de educación artística, principalmente en la asignatura de música. Siempre se ha venido considerando que ésta permite descubrir otras músicas diferentes a la autóctona (Volk, 2004) y que contribuye a garantizar la interculturalidad de la sociedad, porque es un producto cultural en continua transformación (Vidal, Durán y Vilar, 2010).

Sin embargo, para que el alumnado pueda comprender la importancia de la relación-diálogo-intercambio entre culturas, el profesorado debería contar con una competencia en este sentido. Sobre todo si se comprende la competencia docente como la parte normativa del trabajo académico que le permitirá desarrollar su práctica adecuadamente, sea cual sea el contexto (Guzmán y Marín, 20II). El docente tiene que comprender las diferentes opciones educativas relacionadas con la pluriculturalidad, para reflejar adecuadamente su preferencia en los distintos niveles de concreción curricular y en su práctica diaria.

La revisión de propuestas y el análisis de las mismas, ha partido de las siguientes interpretaciones conceptuales recogidas por Bernabé (20I2) y elaboradas a partir de las conclusiones de importantes especialistas en la materia de toda España como Muñoz (200I), García y Escarbajal (2009), tal como pueden observarse en los Cuadros I y 2 propuestos por Bernabé (20I2). Todo educador que trabaje con alumnado de diferentes culturas debería conocer las características y objetivos de cada opción pedagógica, porque sino no podrá aplicar todas sus posibilidades y obtener la situación cultural deseada. 


\section{EDUCACIÓN MULTICULTURAL}

\section{Enfoques}

I. Afirmación cultural hegemónica del país

Modelo asimilacionista:

- Coexistencia sin integración.

- Diversidad cultural comprendida como amenaza a la integración y cohesión social.

- Pérdida de la cultura más débil.

- Minorías deben liberarse de su cultura para no retrasarse académicamente.

\section{Reconocimiento de la pluralidad cultural de acogida}

\section{Modelo de currículum multicultural:}

- Inclusión de elementos culturales de las minorías en currículo educativo.

- Programas biculturales y bilingües.

\section{Modelo segregacionista:}

- Segregación de minorías o grupos raciales.

Separación de culturas en escuelas específicas.

\section{Modelo de orientación multicultural:}

- Desarrolla autoconcienciación cultural para fortalecerla.

- Preserva cultura minoritaria en la nueva sociedad.

\section{Modelo compensatorio:}

- Minoría cultural tiene escasísimo nivel educativo y necesita ayuda para mejorar.

- Minoría rechaza su cultura para defenderse en su nueva sociedad.

\section{Modelo de pluralismo cultural:}

- Respeto y reconocimiento cultural.

- Promueve identificación y pertenencia étnica.

- Atiende a los estilos de aprendizaje y contenidos culturales específicos.

- Promueve el enriquecimiento del contacto y la sensibilidad ante la sociedad pluricultural.

\section{Modelo de competencias multiculturales:}

- Habilidades y actitudes para poder participar activamente en culturas mayoritaria y minoritaria.

Cuadro I, elaborado por Bernabé (20I2) a partir de información citada en Muñoz (200I) 


\section{EDUCACIÓN INTERCULTURAL}

\section{Enfoques}

\section{Integración cultural}

\section{Reconocimiento de la Pluriculturalidad}

3. Simetría cultural

\section{Modelo de educación} antirracista: multicultural:

- Modifica currículo educativo para mejorar éxito escolar de minorías.
- Eliminación del racismo.

- Evita desigualdades y desventajas sociales.

- Respeto y aceptación de diversos grupos culturales presentes en la sociedad.

\section{Modelo de educación no} racista:

- Reducción de actitudes racistas.

- Respeto y aceptación de diversos grupos culturales presentes en la sociedad.

\section{Modelo de orientación} multicultural:

- Desarrollo del autoconcepto cultural.

\section{Modelo holístico:}

- Implicación de toda la escuela en la labor educativa.

- Rechaza el predominio cultural.

- Promueve la igualdad de oportunidades sociales, económicas y educativas.

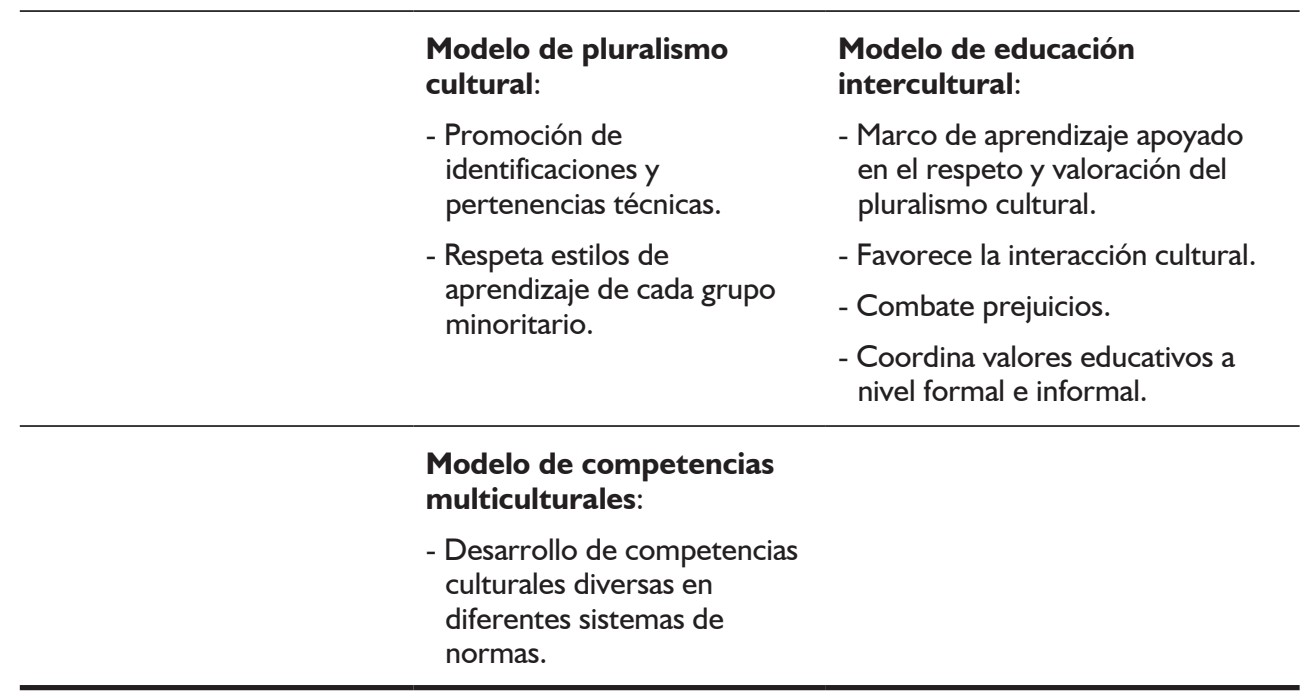

Cuadro 2, elaborado por Bernabé (2012) a partir de información citada en Muñoz (200I) y García y Escarbajal (2009) 
Parece que todavía no ha terminado de comprenderse la pluriculturalidad como una oportunidad para el intercambio-cambio cultural (Louzao, 20l I) y el enriquecimiento mutuo (González, 2008). En este sentido, el aula musical se ha venido utilizando desde la LOGSE como un espacio adecuado para garantizar la consecución de la interculturalidad, entendida como situación ideal que persigue la reconstrucción cultural compartida gracias al diálogo-intercambio. Por ello es tan importante que el docente sea formado para comprender qué opción educativa es más recomendable. Sólo así podrá enfocar su labor docente con la corrección debida para el modelo y enfoque elegido.

\section{La música como herramienta educativa en contextos pluriculturales}

Touriñán y Longueira (2010) plantean que la educación por la música contribuye a educar en valores. Si se parte de la consideración de que la educación musical debe contribuir a la adquisición de las Competencias Básicas, resulta lícito considerarla imprescindible para educar interculturalmente, como se verá en las propuestas analizadas en el posterior epígrafe.

Pero, iqué tiene esta materia diferente a otras presentes en el currículo de la educación obligatoria? ¿Qué elementos la convierten en un instrumento educativo intercultural por excelencia? Seguidamente se profundizará en la importancia de la interpretación, la improvisación y la composición, interpretadas desde su vertiente más cooperativa y comparada para demostrar cómo gracias a ellas el aula musical se convertirá en un espacio educativo que garantice una competencia intercultural (Vilá, 2008), siempre que el docente posea las competencias personales y de valores necesarias para desarrollar su labor didáctica (Zahonero y Martín, 20I2).

Cuando se escucha una pieza musical se puede interpretar de modos muy diversos, pero elementos como las figuras musicales, los signos de expresión, los signos de articulación, los patrones rítmicos, la pueden convertir en un producto cercano a diferentes culturas. Así podría hablarse de un lenguaje universal o que debería percibirse como tal (Campbell, 2005); aunque no todos los expertos comparten esta consideración (Elliott, 1989; Vilá, 2008). En general, se puede decir que la música ofrece un lenguaje accesible a todos los intérpretes porque éstos comparten signos musicales (códigos) que son similares en casi todo el mundo (Pérez y Leganés, 20I2).

Tomar consciencia de los elementos comunes y compartidos es de gran relevancia para la consideración de la música como un medio de comunicación entre culturas. 
No obstante, como podrá comprobarse a través de las distintas experiencias revisadas en el epígrafe siguiente, no bastaría con esa toma de consciencia, sino que haría falta un cambio de perspectiva de "qué comparte él conmigo" a "qué comparto yo con él". Este giro implicaría mayor respeto hacia lo que "el otro" ofrece, cuestión que a nivel de comunicación emocional es de mayor relevancia.

Será la interpretación musical, con su necesidad de la comprensión del material que "el otro" ofrece, la que asiente las bases del diálogo (como intercambio) cultural en el aula educativa. Pero, ante todo la música promueve la sensibilidad como dimensión imprescindible para interiorizar valores universales (Buxarrais y Martínez, 2009), como por ejemplo la tolerancia (Volk, 2004). Así pues resultaría lícito considerar que la música crea actitudes positivas para el proceso educativo intercultural, si ésta se comprende como una actividad participativa y creativa que recoja las manifestaciones culturales autóctonas y del resto del mundo.

\section{Análisis de experiencias musicales en contextos pluriculturales}

La música cuenta con la peculiaridad de que los diferentes elementos culturales que la componen son inseparables e imprescindibles para garantizar la comprensión del conjunto. Tal es esta situación que puede considerarse el producto intercultural por excelencia: las características compositivas son compartidas por diferentes culturas, despierta emociones comunes, posibilita múltiples interpretaciones, y favorece el diálogo a través de la interpretación conjunta. De acuerdo con estas ideas, diferentes investigaciones y experiencias prácticas en Primaria y Secundaria a lo largo de estas primeras décadas del siglo XXI, vienen a corroborar cómo una metodología participativa favorecerá el contacto social y la reflexión cultural, además de que el uso de música moderna con sus elementos claramente fusionados permite reflexionar culturalmente.

Ahora bien, la terminología empleada en ellas es muy diferente. Cada especialista musical hace un uso totalmente distinto de las características de cada enfoque pedagógico relacionado con la diversidad cultural. Es decir, que en muchos casos se habla de interculturalidad pero no se hacen presentes las especificidades de ésta; e incluso en otros se presenta un título multicultural pero es una propuesta intercultural. Todo esto viene a demostrar las carencias formativas de las especialidades musicales, así como la necesidad de revisar la propia práctica docente para cumplir con esos principios de la interculturalidad perseguida en la legislación vigente. 
Se ha realizado un breve análisis de las propuestas más significativas, dentro de todas aquellas que se han revisado y que aparecen catalogadas en el Cuadro 3 que se presenta a continuación. Éste se ha estructurado con la siguiente lógica: dentro de las dos columnas que representan las dos opciones educativas (multicultural e intercultural) citadas en los títulos de los trabajos revisados, se ha realizado una subdivisión que se corresponde con la correcta o incorrecta aplicación del término. Es decir, que si la denominación del título es de "intercultural", pero no se fomenta el intercambio sino sólo el simple conocimiento de otras músicas, estaríamos ante un enfoque multicultural, de acuerdo con las interpretaciones terminológicas ya comentadas en el epígrafe primero.

\begin{tabular}{|c|c|c|c|}
\hline \multicolumn{2}{|c|}{ Denominación “Multicultural” } & \multicolumn{2}{|c|}{ Denominación “Intercultural” } \\
\hline Multicultural & $\begin{array}{l}\text { Realmente } \\
\text { intercultural }\end{array}$ & Intercultural & $\begin{array}{l}\text { Realmente } \\
\text { multicultural }\end{array}$ \\
\hline Giráldez (1997) & & Leiva (2005) & $\begin{array}{c}\text { Gallego y Gallego } \\
\text { (2003) }\end{array}$ \\
\hline Montoro (2002) & & Campos (2006) & Segura y Ortells (2005) \\
\hline Vilar (2006) & & López (2006) & Martínez (2006) \\
\hline $\begin{array}{c}\text { Bravo y De Moya } \\
\text { (2006) }\end{array}$ & & Ruiz (2007) & $\begin{array}{c}\text { Gómez Quintana } \\
\text { (20II) }\end{array}$ \\
\hline \multirow{3}{*}{\multicolumn{2}{|c|}{$\begin{array}{l}\text { Lorenzo y Espejo } \\
\qquad(201 \mathrm{I})\end{array}$}} & Martín (2009) & Escudero (20I2) \\
\hline & & Díez (2009) & $\begin{array}{l}\text { De Moya, Hernández y } \\
\text { Hernández }(2010)\end{array}$ \\
\hline & & Epelde (20II) & González (20।0) \\
\hline
\end{tabular}

Cuadro 3 (elaboración propia)

Abundan los casos en los que el término "multicultural" es asimilado con el de "pluricultural", lo que lleva a que después se hagan referencias a aplicaciones de propuestas interculturales. Éste es el caso de Vilar (2006), que presenta una propuesta multicultural basada en la difusión de música de otras culturas, pero las denomina prácticas interculturales en entornos multiculturales, y choca con las 
consideraciones de los distintos teóricos ya comentados en epígrafes anteriores. En el mismo caso estaría Martín (2009), pero no así Epelde (20l I), quien sí menciona un contexto multicultural pero presenta una intención intercultural.

De Moya, Hernández y Hernández (2010), así como González (2010) defendían una metodología de participación activa desde una propuesta educativa intercultural en Primaria que favorecía el conocimiento "del otro" a través de la canción popular. Desde nuestra experiencia en el aula se ha podido comprobar que este tipo de música resulta lejana a todo el alumnado, autóctono o no, pero es uno de los materiales más utilizados para acercarse a la alteridad como muestra la experiencia de Lorenzo y Espejo (20I I), quienes también recomiendan al profesorado el folklore musical para educar en Primaria y Secundaria.

López (2006) propone prácticas musicales interculturales basadas en el uso de la canción tradicional (aquella transmitida vía oral, de generación en generación), pero con la diferencia de que son los niños de Primaria los que indican sus preferencias musicales de acuerdo con aquellas piezas que hayan escuchado en sus casas. Resulta interesante porque comenta la idea de la fusión de elementos culturales, la incorporación de los aprendidos a los propios. Todo esto se mueve dentro de los intereses y objetivos de la educación intercultural.

Las experiencias interculturales con música no tradicional o no popular son bastante escasas. Algunos autores como Swanwick (2000) consideran que la diversidad cultural va más allá de ese uso de la música popular y tradicional, y defiende la utilización de otras músicas. En esta dirección caminan las propuestas de Bravo y De Moya (2006) que trabajan fragmentos de la BSO de "Los Aristogatos" y canciones de "El Canto del Loco" para educar interculturalmente en el aula de Infantil y Primaria. Aunque, desde nuestra experiencia que se comenta en el epígrafe posterior, lo importante será que el alumnado pueda experimentar con cualquier música e intercambiar ideas con "el otro".

En ocasiones, la variedad en el tratamiento de la educación intercultural musical se limita a la mayor o menor incorporación de competencias a trabajar en las actividades. Un ejemplo de este caso sería Escudero (20I2), que propuso que la interculturalidad se trabaje a través del flamenco, pero incorporando la competencia en nuevas tecnologías para que esta música tradicional resulte más atractiva al alumnado. Así se deja ver la importancia que tiene que el docente sea competente a nivel tecnológico (Alonso, 2012) además de a nivel intercultural. 
Entre el profesorado que desarrolla una actividad educativa intercultural musical alejada de la interpretación o estudio teórico de elementos característicos de otras músicas, destacan las propuestas de composición de Ruiz (2007). La composición es un recurso que permite conocer las diferentes estructuras armónicas y rítmicas, al tiempo que invita a la reflexión de los propios elementos musicales. Este elemento junto con la improvisación musical son de los más utilizados para trabajar la creatividad en el aula. Precisamente serán las actividades creativas las que muestren la diversidad como algo "normal" y común, opuesto a la homogeneización (Díez, 2009), y totalmente en la línea de la educación intercultural como las propuestas propias que se presentan seguidamente.

\section{Una experiencia musical intercultural en Primaria}

La música podrá utilizarse como herramienta educativa intercultural si en su proceso educativo se recurre a propuestas metodológicas activas, participativas y cooperativas (Bernabé, 20I I; Bravo y De Moya, 2006; De Moya, Hernández y Hernández, 2010). Éstas deben partir de un docente con competencia en organización y animación de situaciones de aprendizaje, en gestión de la progresión del aprendizaje, en trabajo en equipo, en organización de la propia formación y en la implicación de los padres (Tejada, 2009). La colaboración de la familia como elemento de unidad es determinante para el desarrollo sociocultural del alumnado (Campbell, 2000; Gómez, 2005).

A continuación se presentan diferentes actividades desarrolladas con alumnado de Educación Primaria. Lo más significativo de éstas es que parten del trabajo de la creatividad del alumnado como principal elemento que garantizará su apertura mental hacia el compañero. Se presentan actividades que implican diálogo, reflexión e intercambio, pero a través de la interpretación musical de composiciones conjuntas como elemento de unión.

Una actividad muy común es la rueda de improvisaciones rítmicas, pero en nuestra clase se incorporaban elementos de música tradicional de otras partes del mundo cuya búsqueda se había solicitado previamente (debían preguntar a sus progenitores). De modo que, una vez presentados esos ritmos y repetidos, se procedió a incorporar nuevos ritmos propios que considerasen podían resultar apropiados como acompañamiento. Para ello tuvieron que elegir un instrumento que no pudo ser el pandero porque era el instrumento de la profesora. El resultado 
fue una fusión y re-construcción compartida de un ritmo "externo" que quedaba incorporado a la microsociedad del aula, con el enriquecimiento intercultural que esto supone.

La composición es más trabajada en Educación Secundaria, como puede verse en la propuesta de Ruiz (2007), aunque sí es posible utilizarla en Primaria y adecuarla para las necesidades educativas interculturales. En nuestro caso, se realizó una adaptación muy simplificada de las normas de composición clásica.

Se eligió un ritmo de entre todo un abanico de diferente procedencia y se dispuso en un pentagrama pero sin las cabezas, sólo las plicas. Después, se colocó una secuencia clásica tipo I-V-I-IV-II-V-I, y en cada uno de esos compases se colocaron las notas del acorde tríada respectivo (tonalidad de Do Mayor); de manera que el alumnado se limitó a seleccionar la nota de su preferencia y a unirla con la plica correspondiente. Quizá la mayor dificultad estribó en que se pusiesen de acuerdo en la selección de las notas, que iban tocando con un carrillón para escuchar el efecto final. Así lo que se estaba consiguiendo era incorporar elementos externos a los suyos, con el consiguiente enriquecimiento cultural que se veía reforzado por el diálogo en torno a los mismos.

Esta actividad compositiva permitió, al mismo tiempo, un trabajo de la improvisación, ya que sobre esa melodía conjunta y sobre un ritmo "extraño" tuvieron que improvisar distintos acompañamientos rítmicos-corporales. Estaban incorporando a su propia producción otros elementos creados por los compañeros, lo que les permitió enriquecerse y ser conscientes de la similitud de propuestas. Precisamente es la similitud de propuestas, a pesar de la diferencia de orígenes, la que lleva a la reflexión cultural y a la comprensión del otro.

Una actividad menos activa pero también muy interesante consistió en la realización de un juego de memoria visual que dio pie a la improvisación de acompañamientos. En distintas tarjetas se escribieron fragmentos musicales de procedencia diversa, que se colocaban boca abajo y cuya pareja debían buscar; ésta era el plano con el nombre del país. La pieza fue interpretada por nosotros y después debían tratar de adivinar el nombre del país de acuerdo con las características trabajadas. Pero no quedó en esto, que podría parecer demasiado multicultural, sino que tras encontrar todas las parejas, se improvisaron acompañamientos individuales que debían ser repetidos por todos mediante turnos. Así se pudieron enriquecer con las aportaciones del compañero e incorporarlas a sus propias producciones. 
En esta línea de trabajo musical creativo que une una formación intercultural para el docente y una colaboración familiar, si se usan la composición y la improvisación, éstas contribuirán a la comprensión "del otro" y al intercambio de experiencias. Precisamente éstos son los principales objetivos de la educación intercultural: la comprensión "del otro" y el intercambio para garantizar la reconstrucción cultural conjunta y compartida.

\section{Conclusiones}

Son muchos los autores que defienden la importancia de la música como transmisora de valores o favorecedora del diálogo y del intercambio (Bernabé, 20 I I; Ros, 2009); todo esto se debe a que el arte musical contribuye a la apertura mental hacia "el otro". Tal como muestran las experiencias personales comentadas, si se recurre a la vertiente más artística y creativa del proceso educativo musical, éste se podrá convertir en vehículo garante de la comunicatividad de los estudiantes. Con el tipo de prácticas planteadas se podrá evitar que el aula se convierta en un bastión de la tradición musical clásica europea que obvie el resto de músicas (Anderson y Campbell, 20II).

La creatividad se convierte en un instrumento de conocimiento intercultural porque es causa y consecuencia del desarrollo humano (Díez, 2009). De entre todas las actividades que favorecen ese desarrollo creativo tan importante para la interculturalidad, la composición es una de las más interesantes por la reflexión y el intercambio cultural que conlleva, tal como se deduce de las propuestas de Ruiz (2007) y de las presentadas en el anterior epígrafe. No obstante, como se habrá podido deducir del análisis realizado, son más comunes las actividades de audición e interpretación.

Todas estas actividades que pueden desarrollarse en el aula de música permiten comprender la importancia de esta materia para que el alumnado consiga una competencia intercultural, muy importante actualmente. No obstante, ya que esta asignatura no es considerada imprescindible como se deduce con las reducciones horarias de cada legislatura, en las manos de los docentes queda el demostrar su gran importancia para la eliminación de las injusticias culturales (Elliott, 2007).

Ante todo, debe evitarse que el alumnado asista pasivo a esta clase de música porque su única posibilidad de interactuar se reduzca a la contestación al docente 
(Vidal, Durán y Vilar, 20I0); es decir que el centro educativo debe modificar los elementos curriculares para posibilitar la igualdad de oportunidades de los educandos.

Pese a lo útiles que puedan resultar las actividades puntuales de jornadas o encuentros, se han quedado obsoletas respecto a las necesidades del nuevo siglo. Ese cambio de unas propuestas multiculturales a otras interculturales ha devenido como resultado de una nueva concepción de la diversidad (Vargas, 2007); aunque el cambio en la formación de los docentes no haya corrido parejo ya que maestros y profesores siguen sin contar con una formación intercultural en sus períodos de formación universitaria (Goenechea, 2008; Jordán, 2007), a lo que Fernández y Malvar (2009) han añadido el miedo de los docentes ante el cambio.

A esta escasez formativa se suma la actitud del alumnado en unas clases que no se adaptan a la realidad pluricultural porque los docentes carecen de competencias para incorporar estrategias interculturales en el aula. Desde nuestra experiencia, en esos momentos de tensión con los educandos se recurría a la transformación de este espacio musical en un punto de experimentación y de vivencia sociocultural; es decir que el proceso educativo se orientaba a un acercamiento al diálogo musical gracias a actividades interactivas socialmente y creativas musicalmente.

En conclusión, si la música es un producto cultural representativo, puede ser una importante herramienta para desarrollar la interculturalidad gracias a una aproximación comparada a sus diferentes elementos.

\section{Referencias bibliográficas}

Alonso, A. (2012). Competencias para docentes y formadores en una sociedad tecnológica. Aularia: Revista Digital de Comunicación, I (2), 247-250.

Anderson, W. M. y Campbell, P. S. (20I I). Teaching music from a multicultural perspective. En W. M. Anderson y P. S. Campbell (Eds.), Multicultural perspectives in music education, (pp. I-7). Plymouth: R\&L Education.

Bernabé, M. (20II). La Educación Intercultural en el aula de Música. Unidades didácticas interculturales para Educación Primaria. Saarbrücken: Editorial Académica Española.

Bernabé, M. (2012). Pluriculturalidad, multiculturalidad e interculturalidad, conocimientos necesarios para la labor docente. Hekademos, II, 67-76. 
Bravo, R. y De Moya, Ma V. (2006). Multiculturalidad musical para las aulas del siglo XXI. Ensayos, 21, I3I-139.

Buxarrais, Ma R. y Martínez, M. (2009). Educación en valores y educación emocional: propuestas para la acción pedagógica. Revista Electrónica Teoría de la Educación. Educación y Cultura en la Sociedad de la Información, 2 (I0), 263-275.

Campbell, A. (2000). Cultural identity as a social construct. Intercultural Education, II (I), 3I-39.

Campbell, P. S. (Ed.) (2005). Cultural diversity in music education. En Cultural diversity in Music Education (pp. VII). Bowen Hills: Australian Academic Press.

Campos, J. L. (2006). Interculturalidad, Identidad y Migración en la Expansión de las Diásporas Musicales. Razón y palabra, 49, 1023.

De Moya, Ma V., Hernández, J. R. y Hernández, J. A. (2010). Experiencia intercultural en el aula "La música nos une". Música y Educación, 84, 18-26.

Díez, P. (2009). Educación artística: lugar de vecindada para el desarrollo humano. Pulso, 32, I23-I45.

Elliott, D. J. (1989). Key concepts in multicultural music education. International Journal of Music Education, I, I I- I8.

Elliott, D. J. (2007). Socializing music education. Action, Criticism, and Theory for Music Education, 6 (4), 60-95.

Epelde, A. (20II). La interculturalidad en la Educación a través de la música infantil. DEDiCA. Revista de educaçao e humanidades, I, 273-292.

Escudero, J. P. (2012). Interculturalidad e integración social en el aula a través del flamenco y los medios audiovisuales. Orientaciones y propuestas didácticas. DEDiCA. Revista de Educaçao e Humanidades, 3, 259-270.

Fernández, Ma D. y Malvar, Ma L. (2009). Una escuela inclusiva para una sociedad plural: nuestro referente, nuestro futuro. XXI. Revista de Educación, II, I83-194.

Gallego, C. I. y Gallego, Ma M. (2003). Interculturalidad en educación infantil y primaria con la música. Filomúsica, 37. Recuperado de http://filomusica.com/filo37/ interculturalidad.html.

García, A. y Escarbajal, A. (Dir.) (2009). Pluralismo sociocultural, educación e interculturalidad. Badajoz: Abecedario. 
Giráldez, A. (1997). Educación musical desde una perspectiva multicultural: diversas aproximaciones. Revista Transcultural de Música, I, I-I 3.

Goenechea, C. (2008). ¿Es la formación del profesorado la clave de la educación intercultural? Revista española de pedagogía, 239, I I9-136.

Gómez Montes, J. M. (2005). Pautas y estrategias para entender y atender la diversidad en el aula. Pulso, 28, 199-2।4.

Gómez Quintana, R. (20II). Música étnica, música intercultural un acercamiento en la Educación Primaria. Sevilla: Asociación por la Innovación Educativa Eduinnova.

González, E. Ma (2008). Convivencia intercultural en centros educativos multiculturales... iHacia un reconocimiento de la diversidad cultural? Educatio Siglo $X X I, 26,225-240$.

González, O. (2010). Una experiencia de curriculum musical intercultural. Música y Educación, 81, 18-33.

Guzmán, I. y Marín, R. (20I I). La competencia y las competencias docentes: reflexiones sobre el concepto y la evaluación. Revista Electrónica Interuniversitaria de Formación del Profesorado, I4 (I), I5I-163.

Jordán, J. A. (2007). Formación intercultural del profesorado de secundaria. Estudios sobre Educación, 12, 59-80.

Leiva, J. J. (2005). Una mirada intercultural en la educación de la música. Filomúsica, 70. Recuperado de http://filomusica.com/filo70/intercultural.html.

López, G. (2006). Música e interculturalidad. Música para compartir en el aula de secundaria. Revista Iberoamericana de Educación, 39 (3), I-7.

Lorenzo, O. y Espejo, J. A. (20I I). Folklore musical en las aulas de primaria y secundaria. Música y Educación, 87, 36-47.

Louzao, $\mathrm{M}^{\mathrm{a}}$ (20II). La comunidad educativa ante la interculturalidad. Aproximación al estudio de la percepción de la misma en una escuela asturiana. Cultura y Educación, 23 (4), 575-588.

Martín, R. (2009). Integración intercultural en el aula de música: conceptos clave. Revista Ibero-Americana de Estudos em Educaçao, 4 (2). Disponible en http://seer. fclar.unesp.br/iberoamericana/article/viewFile/2778/25I4. 
Martínez, J. (2006). La diversidad cultural en el aula de música de secundaria. Reflexiones y propuestas. I Congreso Internacional de Educación en el Mediterráneo ( I I-I 3 de mayo), Palma de Mallorca.

Montoro, Ma P. (2002). La música, una herramienta de entendimiento multicultural en la escuela. Educación y futuro, 7, 6-12.

Muñoz, (200I). Enfoques y modelos de educación multicultural e intercultural. Madrid: Universidad Complutense de Madrid.

Pérez, S. y Leganés, E. N. (2012). La música como herramienta interdisciplinar: un análisis cuantitativo en el aula de Lengua Extranjera de Primaria. Revista de Investigación en Educación, 10 (I), I27-143.

Ros, N. (2009). El lenguaje artístico, la educación y la creación. Revista Iberoamericana de Educación, 33, I-7.

Ruiz, V. J. (2007). Música y diversidad sociocultural en ESO: la composición musical, un punto de encuentro. Valencia: Generalidad Valenciana.

Segura, Ma T. y Ortells, I. (2005). La Educación Musical en el contexto multicultural educativo de Melilla: ejemplificaciones de programaciones interculturales. En J. L. López (Coord.), Experiencias interculturales en Melilla (pp. 4 I-60). Granada: Universidad de Granada y Sindicato Autónomo de Trabajadores de la Enseñanza.

Swanwick, K. (2000). Música, pensamiento y educación. Madrid: Ediciones Morata.

Tejada, J. (2009). Competencias docentes. Profesorado. Revista de currículum y formación del profesorado, I3 (2), I-I5.

Touriñán, J. M. y Longueira, S. (2010). La música como ámbito de educación. Educación "por” la música y educación "para” la música. Teoría de la educación, 22 (2), $|5|-|8|$.

Vargas, J. Ma (2007). La diferencia como valor: hacia una ciudadanía intercultural. Conceptualización de la diversidad cultural e intervención educativa. Profesorado. Revista de currículum y formación del profesorado, 2 (I I). Recuperado de http:// www.ugr.es/-recfpro/revI I 2COL3.pdf.

Vidal, J., Durán, D. y Vilar, M. (2010). Aprendizaje musical con métodos de aprendizaje cooperativo. Cultura y Educación, 22 (3), 363-378. 
Vilá, R. (2008). La competencia comunicativa intercultural en adolescentes. Infancia y aprendizaje, 3I (2), I47-I64.

Vilar, U. (2006). Educación multicultural en el aula de música: un estudio de caso: la ciudad de Pontevedra. Revista de investigación en educación, 3, II5-132.

Volk, T. M. (2004). Music, Education, and Multiculturalism: Foundations and Principles. Oxford: Oxford University Press.

Zahonero, A. y Martín, M. (2012). Formación integral del profesorado: hacia el desarrollo de competencias personales y de valores en los docentes. Tendencias pedagógicas, 20, $5 \mathrm{I}-70$. 